\title{
Los cuestionarios y tablas analíticas de evaluación de las revistas científicas como guía para la iniciación en la escritura científica del alumno de doctorado
}

\author{
José RODRÍGUEZ TERCEÑO \\ Universidad Complutense de Madrid \\ josechavalet@gmail.com
}

Recibido: 04/07/2012

Aceptado: 16/10/2012

\begin{abstract}
Resumen
El alumno recién licenciado puede decantarse por la realización del doctorado, emprendiendo el camino que lo conducirá al mundo de la docencia y la investigación, cuyo primer paso es la realización de una tesis doctoral, su particular tarjeta de presentación dentro de la amplia comunidad científica. La escritura de un trabajo de investigación original supone afrontar y superar varios obstáculos. Las exigencias de acreditación obligan a recurrir al método heurístico 'ensayo-error' o a seguir los consejos que algunas revistas ofrecen a sus autores, con la esperanza de que mediante la práctica se alcance la perfección. En el presente artículo se analizan los puntos más importantes de los cuestionarios y tablas de evaluación de dos de las revistas científicas más punteras en la investigación de comunicación de nuestro país.
\end{abstract}

Palabras clave: Doctorando, tesis, artículo, revista, publicación.

\section{Questionnaires and analytical evaluation boards of scientific journals to guide the doctoral student in the beginning of the scientific writing}

\begin{abstract}
The new graduate student may opt to undertake the doctorate, taking the road that takes him into the world of teaching and research, whose first step is making a doctoral thesis, his particular business card within the scientific community. Writing an original research work involves face and overcome many obstacles. The accreditation requirements obligate to use the heuristic method 'trial and error' or to follow the advice that some magazines offer their authors, with the hope that by practicing they could get perfection. This article discusses the highlights of the questionnaires and evaluation boards of two of the leading scientific magazines in communication research in our country.
\end{abstract}

Keywords: Doctoral student, thesis, article, magazine, publication.

\section{Referencia normalizada}

RODRÍGUEZ TERCEÑO, José (2012): "Los cuestionarios y tablas analíticas de evaluación de las revistas científicas como guía para la iniciación en la escritura científica del alumno de doctorado". Estudios sobre el mensaje periodístico. Vol. 18, núm. especial noviembre, págs.: 781-789. Madrid, Servicio de Publicaciones de la Universidad Complutense.

Sumario: 1. Introducción. 2. La tesis como fuente de artículos científicos. 3. Revistas y artículos. 4. Escritura y revisión. 5. Conclusiones. 6. Referencias bibliográficas.

\section{Introducción}

Pese a las que son y serán las asignaturas pendientes de la universidad española, la enseñanza de la escritura científica -cómo escribir artículos científicos o trabajos de investigación- y la falta de experiencia previa investigadora (en cualquiera de sus ciclos, diplomatura, licenciatura, grado, post-grado, doctorados, máster, etcétera), la carrera científica de cualquier investigador comienza con la elección de cursar el doctorado 
universitario, donde no sólo adquirirá los conocimientos apropiados a su elección de estudio que culminarán con la elaboración de una investigación original en forma de tesis doctoral (un trabajo de investigación que le obligará a invertir en él varios años de su vida, y que será su particular tarjeta de presentación dentro de la amplia comunidad científica una vez defendida y, sobre todo, publicada), sino que además reunirá el saber, teórico y práctico, necesario para la elaboración de artículos científicos cuya publicación contribuirán a la construcción del prestigio del investigador en ciernes.

$\mathrm{La}$ incertidumbre del mundo laboral y de la inexperiencia en la investigación, tanto en su vertiente más práctica -trabajo de campo por mejor decir- como en su lado teórico -realización de investigaciones, escritura científica y publicación de los resultados- marcan el inicio del largo y escabroso camino de todo licenciado que decide dedicarse a la investigación y a la docencia. La inestable salida profesional que puede garantizar cualquier licenciatura o grado, afecta a la decisión de seguir cursando estudios de post-grado; los resultados de una investigación realizada en 2011 entre nuevos alumnos (de comunicación) demostraban que "algo más del $47 \%$ creía estudiar el Grado de Comunicación Audiovisual por vocación, claramente, mientras que otro $40 \%$ respondía que era su vocación con un nivel de convencimiento intermedio [...] descendía considerablemente el grado de optimismo sobre su futuro profesional: menos del 5\% de los encuestados eran muy optimistas respecto a su inserción profesional" (Ortiz Sobrino et al, 2011: web).

La realidad universitaria, víctima constante de las reformas (o contrarreformas) ideológicas de los sucesivos gobiernos, dificultan sobremanera la labor investigadora, especialmente en aquellas ramas no encuadradas en las ciencias puras o exactas, en otras palabras, aquellas cuyas investigaciones doctorales no se desarrollan dentro del laboratorio. "El universo de actividades que implica hacer investigación científica incluye no solo el de identificar elementos de valor que desconocemos y sería valioso conocer (generando hipótesis u objetivos de investigación originales), reconocer $-\mathrm{y}$ producir o conseguir- los materiales y los métodos adecuados para poder poner a prueba esa hipótesis -o satisfacer el objetivo- de modo idóneo, analizar e interpretar los resultados que surgen de la realización del trabajo, sino también hacer que todo lo anterior pueda ser juzgado y eventualmente incorporado a los que llamamos conocimiento científico en el área del conocimiento en la que nos desempeñemos"(Slafer, 2009: web). Afortunadamente, el doctorando puede ejercitarse en el arte de la publicación científica al mismo tiempo que desarrolla su investigación doctoral, no es necesario que espere a que ésta este completada (¿alguna vez puede decirse que un trabajo de investigación doctoral queda completamente terminado?).

Y mientras realiza su investigación el doctorando debe enfrentarse a uno de los retos más complicados dentro del ámbito universitario, la escritura científica, la asignatura pendiente que arrastra la universidad española, "la enseñanza de la escritura científica no se contempla en muchos programas de doctorado" (Rivera-Camino, 2011: 161). Ha de enfrentarse a este reto en estrecha colaboración con su tutor y con otros investigadores con quienes comparte miedos y temores, hasta adquirir una comprensión sistemática y el dominio de las habilidades (métodos de investigación) relacionados con su particular campo de estudio. "Uno de los grandes problemas que 
enfrenta un investigador surge cuando tiene que dar a conocer sus resultados, pues escribir no resulta tarea fácil" (Villagrán y Harris, 2009: 2). Debe, por lo tanto, olvidar los soliloquios que recibió en forma de clase durante sus años de licenciatura y en los seminarios doctorales y, mano a mano con su director de tesis, concebir, diseñar y poner en práctica un proceso de investigación para el que no le han enseñado y para el que debe preparase por su propia cuenta (contando siempre con la colaboración de sus tutores -antiguos alumnos de doctorado y conocedores de las carencias universitarias en este tipo de enseñanzas, la investigación y la escritura científica- y de otros compañeros). En definitiva, debe aprender por cuenta propia algo que la universidad debió facilitarle, cómo afrontar una investigación, desde su gestación hasta su análisis crítico, evaluación, síntesis de las ideas aportadas y publicación de las mismas ya sea como artículos científicos o mediante la tesis doctoral-.

Las exigencias de acreditación para reforzar su currículum le obligan a publicar pronto y con la mayor frecuencia posible, pues mediante las publicaciones se establece en prestigio, credibilidad e impacto de los investigadores. "La publicación es la mejor prueba documental de la capacidad de un investigador para finalizar sus proyectos con éxito [...] un fin intelectual pero, a su vez, es un medio de promoción profesional para quien la suscribe [...] lo más provechoso es publicar pronto y con la mayor frecuencia posible" (Rivera-Camino, 2011: 162). A esta necesidad de publicar cuanto antes se une la falta de experiencia previa en la escritura de artículos de investigación, por lo que el doctorando se ve obligado a recurrir al método heurístico 'ensayo-error' o a seguir los consejos que algunas revistas ofrecen a sus autores, con la esperanza de que mediante la práctica se alcance la perfección. Además de estas breves guías, algunas revistas permiten la visibilidad y descarga de los cuestionarios analíticos y tablas de evaluación, que suponen un inmejorable documento sobre el que apoyarse a la hora de redactar y corregir los artículos científicos postulados para publicación.

\section{La tesis como fuente de artículos científicos}

Ante la falta de clases sobre cómo realizar una investigación y como escribir un artículo científico para su publicación -algo de los que algunos centros se han dado cuenta a tiempo y comienzan a impartir este tipo de clases- no es extraño encontrarnos con artículos en revistas o libros escritos a modo de manual de ayuda entre los alumnos de doctorado, mediante la recopilación de consejos y correcciones de profesores, así como de respuestas negativas y tablas de evaluación que algunas revistas devuelven a sus autores.

No hay que olvidar que el doctorando se enfrenta al aislamiento del escritor, especialmente en el caso de una tesis individual, donde la desconfianza en el trabajo propio, la incertidumbre provocada por la falta de experiencia previa investigadora y, muy especialmente, los juegos malabares que ha de realizar para dedicar tiempo productivo a la redacción de la tesis doctoral reinan por doquier.

A ello hemos de añadir que el doctorando no siempre puede dedicar una jornada completa a la tesis (como debería y como recomiendan cualquiera de los manuales sobre preparación y elaboración de una tesis doctoral o sobre cualquier trabajo de investigación que pueblan el mundo editorial), no siempre puede disfrutar de una beca 
de investigación para realizar su doctorado, y en muchas ocasiones, con el doctorando inmerso en el mundo laboral ajeno a su campo de estudio, tiene que parcelar el tiempo para compaginar trabajo y tesis; $\mathrm{y}$, reconozcámoslo, las más de las veces es ésta última la que sale perdiendo, pues no es fácil enfrascarse cada día en la realización de una tesis doctoral tras el sobreesfuerzo que requiere y exige la ocupación laboral que sustenta económicamente la vida del alumno de doctorado que no disfruta de beca.

Ante esta situación inicial de desconcierto, la falta de experiencia investigadora y de redacción científica (ya fuere para su defensa como para su publicación), la necesidad de publicar mucho y rápido que exige el requerimiento de prestigio investigador y científico a través del impacto de sus artículos o investigaciones, el doctorando aprovecha la realización de su tesis doctoral para, no solo la práctica de la escritura científica, sino también para enfrentarse por primera vez al ciclo de publicación de artículos científicos. Por ello, independientemente de la demostración particular de realizar una investigación original como trabajo final que le conceda el título de doctor, el alumno de doctorado es consciente de la necesidad de extraer de sus tesis uno o varios artículos científicos breves que pueda publicar (o al menos postular para ello), al mismo tiempo que elabora un trabajo cuya estructura no le suponga esfuerzos añadidos en el paso inmediatamente posterior a su defensa, esto es, su publicación. En otras palabras, el doctorando extrae de sus tesis artículos científico mientras redacta la misma, siendo en todo momento consciente de la necesidad de publicar su trabajo una vez ha sido defendido.

\section{Revistas y artículos}

En algunos de los artículos o manuales sobre la publicación de artículos científicos se aconseja seleccionar la revista en la que se quiera publicar antes de trabajar en la redacción del artículo en cuestión. En el caso del doctorando que quiere publicar extractos de su tesis doctoral como artículos de investigación ha de adecuar sus artículos a las revistas en las que tenga intención de publicar, y por ello no sólo es necesario hacer un buen trabajo de adaptación del artículo sino que además se debe estudiar detenidamente algunos factores de las publicaciones antes de seleccionar una (o varias) a las que enviar su artículo (o artículos).

Algunos de estos factores serían:

- Público destinatario al que se dirige la revista, dentro del ámbito de investigación en la que el doctorando se está examinando y en el que espera acabar construyendo su carrera académica o investigadora.

- Prestigio de la publicación, que viene determinado por los índices, bases de datos y directorios de prestigio como pudieran ser, por citar solo algunos: ISI-Wok, Scopus, IN-RECS, DICE, CIRC, Redalyc, Latindex Catálogo, ISOC, EBSO, DIALNET, SciELO, etcétera.

- Acceso, es decir, la accesibilidad para postular publicaciones, si el doctorando puede publicar o se requiere la titulación.

- Impacto, el factor de impacto es el principal instrumento de estructuración de las revistas y de la repercusión entre los investigadores, en otras palabras, cómo se valora nuestro trabajo y cómo se valora a su autor. 
- Periodicidad de la revista, las revista con un carácter mensual o trimestral presentan mejores posibilidades de publicación que una semestral o anual. Por añadidura, las publicaciones con una periodicidad mayor presentan unas exigencias mayores que pueden hacernos revisar el trabajo enviado y no tener la posibilidad de publicación hasta el siguiente número seis o incluso un año después. Las revisiones en una revista de carácter trimestral, por ejemplo, aunque igual de minuciosas, nos permiten poder presentar el artículo corregido en el siguiente número sin que la espera sea muy prolongada.

- Calidad de la revista, es decir, consideración de las características más técnicas de la publicación. Es una revista en papel o electrónica, en color o blanco y negro, permite la inclusión en el artículo de gráficos, imágenes, etc., qué calidad tendrían éstas en la publicación final. Ver en definitiva algunos artículos ya publicados por la revista para tener una 'vista previa' del resultado final de nuestro artículo y saber si de adapta a las necesidades de nuestro trabajo.

- Aceptación: el doctorando debe ser consciente, como señala Germano (2008), que la tesis doctoral es más el último trabajo como estudiante de una persona que su primer trabajo como profesional académico, por ello ha de ser consciente de sus limitaciones iníciales a la hora de seleccionar la revista en la que quiere publicar, tener muy en consideración los requisitos y las evaluaciones de las revistas a las que postula publicación, tanto los criterios de evaluación como las correcciones o razones de rechazo que le fueran enviadas, y sobre todo, no dejar de intentarlo una y otra vez, pues, como ya hemos señalado con anterioridad, solo con la práctica se alcanza la perfección, solo escribiendo y postulando se consigue finalmente publicar.

En definitiva, el doctorando ha de ser minucioso en el proceso de selección de la revista o revistas en las que desea publicar, o al menos a las que desea enviar su trabajo con la intención de que éste vea la luz al final del túnel. Como aconseja RiveraCamino: "Decida con antelación; por lo menos antes de escribir el borrador del artículo. No busque la revista después de haber redactado el artículo. Examine revistas científicas que hayan publicado trabajos similares al suyo. Tome en consideración aquellas revistas científicas que hayan publicado trabajos que hayan sido citados por usted" (Rivera-Camino, 2011: 163).

El propio Rivera-Camino señala la necesidad de preguntarse acerca de la revista en la que el doctorando pretende publicar: ¿Qué categoría de artículos publica la revista? ¿Cuál es la máxima extensión de los artículos? ¿Y de los resúmenes o 'abstracts'?. ¿Tiene la revista plantilla? Puede tenerla tanto para su estructura técnica, forma de presentación del artículo escrito, como para su contenido, división de las diferentes parte que deben constar en un artículo de investigación, como pudieran ser: resumen, palabras clave, introducción, metodología, análisis, desarrollo, resultados, discusión, conclusiones, bibliografía, etcétera. Estás partes también siguen, por lo general, pautas concretas para su redacción.

En definitiva, el doctorando ha de conocer con minuciosidad las exigencias y requisitos que las revistas imponen a quienes pretenden publicar en ellas. El esfuerzo que 
supone este pequeña pero intensa investigación tiene su recompensa gracias al factor de impacto de la revista (véase Castillo Esparcia, 2012), y por ende, del propio autor una vez ha publicado en la comunicación en cuestión.

\section{Escritura y revisión}

La exigencia de estructuración de un artículo científico por parte de una revista vendrá caracterizada por la necesidad de dividir nuestra comunicación en diferentes partes (en algunas revistas más, en otras menos -éstas agrupan en una sola parte lo que otras dividen), pero siempre se ha de incluir: el título, un resumen unido a una selección de palabras clave (entre cinco y siete), una introducción, análisis y discusión del objeto de nuestro estudio, aquí podemos encontrarnos con pequeñas partes que destacan los materiales y los métodos empleados, los resultados obtenidos y discusión de los mismos, y, finalmente, conclusiones y bibliografia empleada. En algunas publicaciones se incluyen agradecimientos $\mathrm{y}$, siempre que no se puedan incorporar al texto, notas, tablas y figuras al finalizar el artículo.

El título de una comunicación, al contrario que las conclusiones, se ha de escribir al finalizar nuestro trabajo (aunque se puede contar con un título provisional), y debe ser conciso, lógico, transparente, preciso y descriptivo. El resumen es la carta de presentación, a partir de éste el lector (la comunidad científica) decidirá si continua leyendo el artículo. Debe suponer una síntesis de cada estructura del artículo, incluir por tanto, aunque de forma muy breve, cuál es el objeto de nuestro estudio, cómo lo hemos estudiado, qué metodología hemos empleado y qué conclusiones hemos obtenido. Es conveniente escribirlo al finalizar en trabajo y siempre de forma impersonal y en pasado. Junto a las palabras clave seleccionadas, el título y el resumen han de despertar el interés del lector lo suficiente como para que continúe leyendo nuestro trabajo. La introducción debe responder a varias preguntas: cuál es el problema y qué se ha escrito sobre él hasta el momento; qué hipótesis o enfoque nuevo introducimos con nuestro trabajo, es decir, cuál es el nuevo aporte al problema abordado.

El análisis, la presentación de los resultados y la discusión, deben ser coherentes, claros y precisos. Si introducimos tablas o gráficos, no se debe repetir la información, sino comentar la conclusión que se extrae de ellos. Tiene que servir para comparar los resultados de nuestra investigación con otros similares ya expuestos por la comunidad científica, qué limitaciones han marcado nuestra investigación o identificar qué errores metodológicos han podido surgir al realizar nuestro estudio, qué relaciones, consecuencias y generalidades indican los resultados y conclusiones que hemos obtenido, así como las nuevas vías de investigación o las necesidades de investigar en el futuro que se abren tras nuestro trabajo y su publicación. "Aquella investigación que ofrezca unos buenos resultados y una buena discusión se asegura su publicación” (Villagrán y Harris, 2009: 5). Las conclusiones deben cerrar lo expuesto anteriormente sin repetirse. Deben ser consecuentes con lo expuesto en el análisis y los resultados obtenidos, así como estar directamente relacionadas con el objeto de estudio y nuestros objetivos.

No debe el doctorando olvidarse de revisar el trabajo antes de enviarlo a la revista científica. En este sentido, son varias las preguntas que debe hacerse, entre otras: ¿Supone nuestro trabajo una contribución original y útil para la comunidad científica? ¿Es 
apropiado para la revista elegida por su temática y por sus lectores potenciales? ¿Se cumplen las normas e instrucciones para la preparación y envío de los trabajos? ¿La metodología que empleamos es la adecuada? ¿Son nuestros análisis, resultados, conclusiones, tablas y figuras lo suficientemente claros y precisos?. ¿Está correctamente estructurado nuestro artículo?. ¿La bibliografía citada cumple las normas? ¿Es actual?. ¿Se corresponden las conclusiones con los objetivos marcados al inicio del trabajo?. ¿Está el resumen y el análisis escrito en pasado?. ¿Y la introducción y las conclusiones en presente?. Son estas y otras muchas preguntas las que debe responder todo doctorando que quiere postular a publicar su trabajo en una revista científica con la que comenzar a tener impacto y se citado dentro de la comunidad científica.

Igualmente, el doctorando puede tener en cuenta los cuestionarios analíticos, las tablas de evaluación de las revistas elegidas, así como los informes de revisión que le fueran enviados en caso de no ser aceptado su trabajo. Un cuestionario analítico para la selección de un artículo de una revista científica (a través de una lectura ciega entre pares) es una valoración realizada por expertos en la materia para comprobar la adecuación de la comunicación a la revista que éste ha elegido para publicar. Se tienen muy en cuenta la acomodación del artículo al ámbito temático de la revista y el cumplimiento de la normativa editorial para la presentación del artículo. También se valora la originalidad, la contribución al conocimiento, la metodología y estructura, la redacción, la relevancia del tema y la calidad de la discusión o las conclusiones alcanzadas en el estudio. La valoración puede ir desde 'muy bien' o 'notable', 'bien', 'suficiente', hasta 'deficiente' o 'pobre'. En el caso de no cumplir o de mostrar unas características insuficientes, el experto en cuestión debe expresar sus razones o sugerencias de corrección para la publicación. Si el resultado del cuestionario es favorable el artículo se publica, si es desfavorable se remite el informe al articulista para que efectúe las correcciones pertinentes y pueda publicarse posteriormente.

Los cuestionarios, así como los informes que algunos investigadores y doctorandos han recibido tras enviar sus trabajos publicables a las revistas elegidas, son una buena guía a tener en cuenta a la hora de redactar el artículo científico y, especialmente, a la hora de corregirlo y revisarlo antes de enviarlo a la revista en cuestión. Seguir este tipo de indicaciones supone tener parte del camino andado a la hora de comenzar a publicar. No obstante, como ya hemos señalado al comienzo de estas líneas, la perfección se alcanza mediante la práctica, por lo que el doctorando tendrá que escribir y reescribir sus artículos una y otras vez hasta alcanzar su objetivo y, por encima de todo, no darse por vencido, pues, como ya hemos señalado la publicación es la presentación del autor dentro de la comunidad científica, a través de esta se puede obtener el factor de impacto que gobierna la calidad del investigador y de las investigaciones en las que participa. Como nos aconseja la Revista de Comunicación Vivat Academia, "publico, luego existo, y si no publico, insisto".

\section{Conclusiones}

El doctorando que establece como uno de sus objetivos principales la publicación científica en revistas de prestigio al tiempo que se enfrenta a la ardua tarea de investigar y escribir su tesis doctoral, sabe que, en líneas generales, este es un camino que 
ha de recorrer solo, pues, como ya hemos visto, la escritura científica -la investigación científica siquiera- es una asignatura pendiente en la universidad española.

Cuenta con apoyos, cierto; los consejos de sus tutores, el apoyo fraternal de otros compañeros investigadores doctorandos enfrentados a las mismas miserias (incertidumbre, soledad del escritor, y demás temores) que él, las recomendaciones que puede encontrar en libros de apoyo para investigar o redactar trabajos científicos, o en artículos sobre redacción publicados por revistas de prestigio, los útiles consejos que ofrecen las revistas en las que se pretende postular una comunicación para su publicación, así como las hojas de evaluación (con sus cuestionarios analíticos y tablas de valoración) que estás muestran a los potenciales autores, y, especialmente, los informes con el resultado de su intencionalidad de publicación, los cuales, en caso de ser negativos, vienen acompañados de observaciones que el doctorando ha de corregir, marcándole el camino en la redacción científica.

La inexperiencia y los temores iniciales del doctorando han de dejar paso al trabajo duro y al esfuerzo continuado que supone labrarse una carrera científica. Una vez iniciada su carrera investigadora, académica y docente con la presentación de su tesis doctoral, el alumno ya convertido en doctor no puede dejar de escribir ni dejar de insistir en publicar. No sólo por necesidades curriculares, sino porque su calidad y su prestigio como investigador científico vienen dados por sus publicaciones, que no son otra cosa que su contribución, mediante pequeños granos de arena que son sus trabajos, a la comunidad científica, al vasto universo que es la ciencia.

\section{Referencias bibliográficas}

CASTILLO ESPARCIA, Antonio (2012): "Investigación e investigadores. Las revistas científicas como instrumentos de comunicación", en Revista de Comunicación Vivat Academia: http://www.ucm.es/info/vivataca/numeros/n117E/PDFs/ACastil.pdf. [Consultado el 20 de julio de 2012]

GERMANO, William (2008): Cómo transformar tu tesis en un libro. Madrid, Siglo XXI.

ORTIZ SOBRINO, Miguel Ángel; RODRÍGUEZ BARBA, Dolores y PÉREZ SERRANO, María José (2011): "Perfil de entrada de los futuros alumnos de comunicación en las universidades de Madrid, en 2010/2012: la reafirmación de los rasgos", en Revista de Comunicación SEECI: http://www.ucm.es/info/seeci/Numeros/Numero 26/DATOS.html. [Consultado el 20 de julio de 2012]

RIVERA-CAMINO, Jaime (2011): Cómo escribir y publicar una tesis doctoral. Madrid, ESIC.

SLAFER, Gustavo A. (2009): “CCómo escribir un artículo científico?”, en Revista de Investigación en Educación: http://webs.uvigo.es/reined/. [Consultado el 20 de julio de 2012]

UNESCO (1983): Guía para la redacción de artículos científicos destinados a la publicación: http://unesdoc.unesco.org/images/0005/000557/055778SB.pdf. [Consultado el 20 de julio de 2012] 
VILLAGRÁN, Andrea y HARRIS, Paul R. (2009): “Algunas claves para escribir correctamente un artículo científico", en Revista Chilena de Pediatría: http://www.scielo.cl/pdf/rcp/v80n1/art10.pdf. [Consultado el 20 de julio de 2012]

\section{José RODRÍGUEZ TERCEÑO}

Universidad Complutense de Madrid. Doctorando josechavalet@gmail.com 